\title{
NCOA4-RET and TRIM27-RET are Characteristic Gene Fusions in Salivary Intraductal Carcinoma, Including Invasive and Metastatic Tumors: Is "Intraductal" Correct?
}

Skálová, Alena ; Ptáková, Nikola ; Santana, Thalita ; Agaimy, Abbas ; Ihrler, Stephan ; Uro-Coste, Emmanuelle ; Thompson, Lester D R ; Bishop, Justin A ; Baněčkova, Martina ; Rupp, Niels J ; Morbini, Patrizia ; de Sanctis, Stefano ; Schiavo-Lena, Marco ; Vanecek, Tomas ; Michal, Michal ; Leivo, Ilmo

\begin{abstract}
Intraductal carcinoma (IC) is the new WHO designation for tumors previously encompassed by "low-grade cribriform cystadenocarcinoma" and "low-grade salivary duct carcinoma." The relationship of IC to salivary duct carcinoma (SDC) is controversial, even though they are considered to be distinct entities. IC is a rare low-grade malignant salivary gland neoplasm with histopathological features reminiscent of atypical ductal hyperplasia or ductal carcinoma in situ of the breast, showing diffuse S100 protein and mammaglobin positivity, while it is partially defined genetically. Recently, RET rearrangements including NCOA4-RET and TRIM27-RET have been described in IC. Here, we genetically characterize the largest cohort of IC to date (33 cases) including 8 cases with focal or widespread invasive growth and 1 case with lymph node metastasis. Thirty-three cases of IC were analyzed by next-generation sequencing (NGS) using the FusionPlex Solid Tumor kit (ArcherDX). Identified gene fusions were confirmed using fluorescence in situ hybridization break-apart and fusion probes and an reverse transcription polymerase chain reaction designed specifically for the detected breakpoints. Ten cases of SDC were analyzed for comparison using NGS panels that detect mutations and fusion transcripts. NGS analysis detected an NCOA4-RET fusion transcript in 11 cases of intercalated duct-type IC joining exon 7 or 8 of NCOA4 gene and exon 12 of the RET gene. Eight cases of IC had an invasive growth pattern, including one with widespread invasion and lymph node metastasis. Three invasive ICs harbored an NCOA4-RET fusion transcript, while 1 case was negative, and 2 cases were not analyzable. In addition, a novel TRIM27RET fusion transcript between exon 3 of TRIM27 and exon 12 of RET was identified in 2 cases of IC with apocrine features, and one of them displayed invasive growth. Two IC cases with invasive growth harbored novel fusions TUT1-ETV5 and KIAA1217-RET, respectively. A total of $42.4 \%$ of the cases in this series of IC harbored fusions involving RET. Such fusion transcripts were not detected in any of the 10 SDC cases. We have confirmed NCOA4-RET as a predominant fusion in intercalated duct-type IC, including 3 cases with invasive growth pattern. A novel finding in our series was a case of widely invasive intercalated duct-type IC, with a single lymph node metastasis that revealed an NCOA4-RET fusion transcript. We also demonstrated that a subset of apocrine ICs harbored a TRIM27-RET gene fusion, including one case with invasive growth. In contrast, neither NCOA4-RET nor TRIM27-RET fusions were detected in any tested SDCs. Thus, the distinct molecular findings in IC and SDC support that the tumors are separate malignant salivary tumor entities. The presence of tumor-type-specific NCOA4-RET or TRIM27-RET translocations in a subset of widely invasive carcinomas with intercalated duct-like immunoprofiles suggests that a recharacterization of IC including its redesignation as "intercalated duct carcinoma, invasive or noninvasive" may be appropriate.
\end{abstract}

DOI: https://doi.org/10.1097/PAS.0000000000001301 
Posted at the Zurich Open Repository and Archive, University of Zurich

ZORA URL: https://doi.org/10.5167/uzh-171765

Journal Article

Originally published at:

Skálová, Alena; Ptáková, Nikola; Santana, Thalita; Agaimy, Abbas; Ihrler, Stephan; Uro-Coste, Emmanuelle; Thompson, Lester D R; Bishop, Justin A; Baněčkova, Martina; Rupp, Niels J; Morbini, Patrizia; de Sanctis, Stefano; Schiavo-Lena, Marco; Vanecek, Tomas; Michal, Michal; Leivo, Ilmo (2019). NCOA4-RET and TRIM27-RET are Characteristic Gene Fusions in Salivary Intraductal Carcinoma, Including Invasive and Metastatic Tumors: Is "Intraductal" Correct? American Journal of Surgical Pathology, 43(10):1303-1313.

DOI: https://doi.org/10.1097/PAS.0000000000001301 


\title{
NCOA4-RET and TRIM27-RET are Characteristic Gene Fusions in Salivary Intraductal Carcinoma, Including Invasive and Metastatic Tumors
}

Is "Intraductal" Correct?

\author{
Alena Skálová, MD, PhD, *广 Nikola Ptáková, MSc, ł Thalita Santana, DDS, MSc, PhD, $\S$ \\ Abbas Agaimy, MD, PhD,\| Stephan Ihrler, MD, PhD, $\mid$ Emmanuelle Uro-Coste, MD, PhD,\#** \\ Lester D.R Thompson, MD, †† Justin A. Bishop, MD, PhD, $\neq \neq$ Martina Baněčkova, MD, * \\ Niels J. Rupp, MD, $\S \S$ Patrizia Morbini, MD,\|\| Stefano de Sanctis, MD, PhD, $\uparrow \uparrow$ \\ Marco Schiavo-Lena, MD,\#\# Tomas Vanecek, PhD, $\neq$ Michal Michal, MD, * \\ and Ilmo Leivo, MD, PhD***
}

\begin{abstract}
Intraductal carcinoma (IC) is the new WHO designation for tumors previously encompassed by "low-grade cribriform cystadenocarcinoma" and "low-grade salivary duct carcinoma." The relationship of IC to salivary duct carcinoma (SDC) is controversial, even though they are considered to be distinct entities. IC is a rare low-grade malignant salivary gland neoplasm with histopathological features reminiscent of atypical ductal hyperplasia or ductal
\end{abstract}

From the *Department of Pathology, Charles University, Faculty of Medicine in Plzen; †Biopticka Laboratory Ltd; \$Molecular and Genetic Laboratory, Biopticka Laboratory Ltd, Plzen, Czech Republic; §Department of Oral Pathology, Faculty of Dentistry, University of São Paulo, São Paulo, Brasil; \|Department of Pathology, University of Erlangen, Erlangen; $\uparrow$ Dermpath, Muenchen, Germany; \#Department of Pathology, Toulouse University Hospital, IUCOncopole; **INSERM U1037, Cancer Research Center of Toulouse (CRCT), Toulouse, France; ††Department of Pathology, Southern California Permanente Medical Group, Woodland Hills, CA; $\$$ Department of Pathology, UT Southwestern Medical Center, Dallas, TX; §§Department of Pathology and Molecular Pathology, University Hospital and University of Zurich, Zurich, Switzerland; \|||Unit of Pathology, University of Pavia and Foundation I.R.C.C.S Policlinico San Matteo, Pavia, Italy; qqHistopathology Department, Addenbrooke Hospital, Cambridge University Hospitals NHS Trust, Cambridge, UK; \#\#Department of Pathology, IRCCS San Raffaele Scientific Institute, Milan, Italy; and ***Institute of Biomedicine, Pathology, University of Turku, and Turku University Hospital, Turku, Finland.

Preliminary results of the study were presented as a platform presentation at the 107th Annual Meeting of the USCAP, Vancouver, Canada, March 17-22, 2018.

Conflicts of Interest and Source of Funding: Supported in parts by the grant SVV-2018 No. 260391 provided by the Ministry of Education Youth and Sports of the Czech Republic (A.S. and M.B.); and by grants from the Finnish Cancer Society and Finska Läkaresällskapet, Helsinki (I.L.). The authors have disclosed that they have no significant relationships with, or financial interest in, any commercial companies pertaining to this article.

Correspondence: Alena Skálová, MD, PhD, Sikl's Department of Pathology, Medical Faculty of Charles University, Faculty Hospital, E. Benese 13, Plzen 305 99, Czech Republic (e-mail: skalova@fnplzen.cz). Copyright (C) 2019 Wolters Kluwer Health, Inc. All rights reserved. carcinoma in situ of the breast, showing diffuse S100 protein and mammaglobin positivity, while it is partially defined genetically. Recently, RET rearrangements including NCOA4-RET and $T R I M 27-R E T$ have been described in IC. Here, we genetically characterize the largest cohort of IC to date (33 cases) including 8 cases with focal or widespread invasive growth and 1 case with lymph node metastasis. Thirty-three cases of IC were analyzed by next-generation sequencing (NGS) using the FusionPlex Solid Tumor kit (ArcherDX). Identified gene fusions were confirmed using fluorescence in situ hybridization break-apart and fusion probes and an reverse transcription polymerase chain reaction designed specifically for the detected breakpoints. Ten cases of SDC were analyzed for comparison using NGS panels that detect mutations and fusion transcripts. NGS analysis detected an NCOA4-RET fusion transcript in 11 cases of intercalated ducttype IC joining exon 7 or 8 of NCOA4 gene and exon 12 of the $R E T$ gene. Eight cases of IC had an invasive growth pattern, including one with widespread invasion and lymph node metastasis. Three invasive ICs harbored an NCOA4-RET fusion transcript, while 1 case was negative, and 2 cases were not analyzable. In addition, a novel TRIM27-RET fusion transcript between exon 3 of TRIM27 and exon 12 of RET was identified in 2 cases of IC with apocrine features, and one of them displayed invasive growth. Two IC cases with invasive growth harbored novel fusions TUT1-ETV5 and KIAA1217-RET, respectively. A total of $42.4 \%$ of the cases in this series of IC harbored fusions involving $R E T$. Such fusion transcripts were not detected in any of the 10 SDC cases. We have confirmed NCOA4-RET as a predominant fusion in intercalated duct-type IC, including 3 cases with invasive growth pattern. A novel finding in our series was a case of widely invasive intercalated duct-type IC, with a single lymph node metastasis that revealed an NCOA4-RET fusion transcript. We also demonstrated that a subset of apocrine ICs harbored a TRIM27-RET gene fusion, including one case with invasive growth. In contrast, neither NCOA4-RET nor TRIM27-RET fusions were detected in any tested SDCs. Thus, the distinct molecular findings in IC and SDC support that the 
tumors are separate malignant salivary tumor entities. The presence of tumor-type-specific NCOA4-RET or TRIM27-RET translocations in a subset of widely invasive carcinomas with intercalated duct-like immunoprofiles suggests that a recharacterization of IC including its redesignation as "intercalated duct carcinoma, invasive or noninvasive" may be appropriate.

Key Words: salivary gland neoplasms, intraductal carcinoma, intercalated duct carcinoma, apocrine, NCOA4-RET, TRIM27-RET, RET-targeted therapy, gene fusion, cohort studies

(Am J Surg Pathol 2019;00:000-000)

n the 2017 World Health Organization Classification of Head and Neck Tumours, ${ }^{1}$ the tumor entity originally described as "low-grade salivary duct carcinoma" 2 and later called "low-grade cribriform cystadenocarcinoma" 3 was renamed as intraductal carcinoma (IC). IC is a rare low-grade salivary gland malignancy with histomorphologic features reminiscent of atypical ductal hyperplasia or ductal carcinoma in situ of the breast. The tumor is, in typical cases, characterized by intraductal and intracystic proliferation of luminal ductal cells exhibiting solid, cribriform, and papillary patterns. Its in situ intraductal nature is demonstrated by an intact myoepithelial cell layer highlighted by antibodies to $\mathrm{p} 63$ protein, calponin, and/or cytokeratin 14. IC typically shows an intercalated duct phenotype demonstrating S100 protein and SOX10 positivity of luminal cells, while a subset of IC shows apocrine morphology further supported by androgen receptor (AR) immunoexpression. ${ }^{4}$ Rare lesions show mixed features of the 2 (hybrid types).

Recent studies investigating the molecular genetics of IC showed recurrent rearrangements of the RET gene with a predominant NCOA4-RET fusion in the intercalated duct-type IC, 5,6 and a TRIM27-RET fusion in the apocrine-type IC ${ }^{6,7}$ Recurrent rearrangements involving RET have been identified in $47 \%$ of cases of low-grade IC with S100 positivity and intercalated duct phenotype. ${ }^{5,6}$ NCOA4-RET fusion was described in noninvasive IC, and the $R E T$ gene was identified as an apparent early oncogenic driver. ${ }^{5,6}$ Interestingly, rare but well-documented cases of IC with focal or widespread invasive growth have been reported. ${ }^{4,6}$ Although previous cases of IC with microinvasion were known, ${ }^{2,8,9}$ the first case of S100 proteinpositive and AR-negative intercalated duct-type IC with NCOA4-RET gene fusion and an invasive component was described recently by Weinreb et al (case 1). ${ }^{5}$ In our previous study, we reported invasive growth in one case of IC with apocrine features and TRIM27-RET gene fusion (case 3). ${ }^{6}$ Thus, there is a growing body of evidence indicating that $R E T$-rearranged ICs are not always indolent in situ malignancies, but may be widely invasive.

Moreover, RET-rearranged IC cases with hybrid intercalated duct and apocrine features have been reported. ${ }^{5,7}$ These findings, in particular, intercalated predominant and pure apocrine carcinomas with widely invasive growth patterns and RET rearrangement suggest that the current term "intraductal carcinoma" (WHO 2017) ${ }^{1}$ should be replaced by a more appropriate designation. It is the opinion of the authors of this study that the term "intraductal carcinoma" is a misnomer because of cases with widely invasive carcinomas with a background of in situ intraductal low-grade lesions harboring NCA4-RET or TRIM27-RET gene fusions. To support this hypothesis, we have genetically characterized the largest cohort of IC to date (33 cases), including 8 cases with focal to widespread invasive growth and 1 case with lymph node metastasis. The relationship of IC to salivary duct carcinoma (SDC) has been controversial since the first description of IC..$^{2,8-13}$ Ten cases of SDC were analyzed for comparison using next-generation sequencing (NGS) panels that detect mutations and fusion transcripts.

\section{MATERIALS AND METHODS}

Among $>6200$ cases of primary salivary gland tumors, 33 cases of IC were retrieved from the consultation files of the Salivary Gland Tumor Registry, at the Department of Pathology, Faculty of Medicine in Plzen, and Biopticka Laboratory Ltd, Plzen, Czech Republic, and from the consultation files of the coauthors. Twenty-nine cases originally diagnosed as SDC were, for comparison, retrieved from the consultation files of the Salivary Gland Tumor Registry, and 10 of them were analyzed by NGS.

The histopathologic features of all tumors and the immunohistochemical stains, when available, were reviewed by 3 pathologists (A.S., T.S., and M.B.). A diagnosis of IC was confirmed in cases that displayed histologic features consistent with the original description in conjunction with the appropriate immunohistochemical profile, that is, co-expression of S100 protein, cytokeratin CK7, and mammaglobin in the absence of DOG1 staining. Moreover, an intact myoepithelial layer decorated by p63 and/or CK14 and calponin provided evidence of the intraductal component. Tumors were classified further into 2 groups, according to criteria published recently ${ }^{4}$ : those that showed an intercalated duct phenotype and those with an apocrine phenotype. This was determined both histologically and immunohistochemically. Thus, a total of 33 IC cases were studied by NGS using ArcherDX Fusion Plex kit.

A diagnosis of SDC was confirmed in cases that displayed histologic features consistent with original description in conjunction with the appropriate immunohistochemical profile, using criteria published recently. ${ }^{14}$

For conventional microscopy, the excised tissues were fixed in formalin, routinely processed, embedded in paraffin (FFPE), cut, and stained with hematoxylin and eosin.

For immunohistochemical analysis, 4- $\mu$ m-thick sections were cut from paraffin blocks and mounted on positively charged slides (TOMO, Matsunami Glass IND, Japan). Sections were processed on a BenchMark ULTRA (Ventana Medical System, Tucson, AZ), deparaffinized, and then subjected to heat-induced epitope retrieval by immersion in a $\mathrm{CCl}$ solution at $\mathrm{pH} 8.6$ at $95^{\circ} \mathrm{C}$. All primary 


\begin{tabular}{|c|c|c|c|c|}
\hline $\begin{array}{l}\text { Antibody } \\
\text { Specificity }\end{array}$ & Clone & Dilution & $\begin{array}{c}\text { Antigen } \\
\text { Retrieval/time }\end{array}$ & Source \\
\hline S-100 protein & Polyclonal & RTU & $\mathrm{CC} 1 / 20 \mathrm{~min}$ & Ventana \\
\hline Mammaglobin & $304-1 \mathrm{~A} 5$ & RTU & $\mathrm{CC} 1 / 36 \mathrm{~min}$ & DakoCytomation \\
\hline CK7 & $\begin{array}{c}\text { OV-TL } \\
12 / 30\end{array}$ & $1: 200$ & $\mathrm{CC} 1 / 36 \mathrm{~min}$ & DakoCytomation \\
\hline p63 & $4 \mathrm{~A} 4$ & RTU & $\mathrm{CC} 1 / 64 \mathrm{~min}$ & Ventana \\
\hline SOX-10 & Polyclonal & $1: 100$ & $\mathrm{CC} 1 / 64 \mathrm{~min}$ & Cell Marque \\
\hline MIB1 & $30-9$ & RTU & $\mathrm{CC} 1 / 64 \mathrm{~min}$ & Ventana \\
\hline $\begin{array}{l}\text { Androgen } \\
\text { receptor }\end{array}$ & SP107 & RTU & $\mathrm{CC} 1 / 64 \mathrm{~min}$ & Cell Marque \\
\hline Calponin & EP798Y & RTU & $\mathrm{CC} 1 / 36 \mathrm{~min}$ & Cell Marque \\
\hline CK 14 & SP53 & RTU & $\mathrm{CC} 1 / 64 \mathrm{~min}$ & Cell Marque \\
\hline
\end{tabular}

$\mathrm{CC} 1$ indicates EDTA buffer, pH 8.6; RTU, ready to use.

antibodies used are summarized in Table 1. The bound antibodies were visualized using the ultraView Universal DAB Detection Kit (Roche) and ultraView Universal Alkaline Phosphatase Red Detection Kit (Roche). The slides were counterstained with Mayer's hematoxylin. Appropriate positive and negative controls were used.

Where available, clinical follow-up was obtained from the patients, their physicians, or from referring pathologists.

\section{Molecular Genetic Study}

\section{Sample Preparation for NGS and Reverse Transcription Polymerase Chain Reaction}

For NGS and reverse transcription polymerase chain reaction (RT-PCR) analysis, 2 to 3 FFPE sections (10 $\mu \mathrm{m}$ thick) were macrodissected to isolate tumor-rich regions. Samples were extracted for total nucleic acid using Agencourt FormaPure Kit (Beckman Coulter, Brea, CA). The RNA integrity was evaluated using PreSeq RNA QC Assay, as was previously described. ${ }^{6}$

\section{RNA Integrity Assessment and Library Preparation for NGS}

Unless otherwise indicated, $250 \mathrm{ng}$ of FFPE RNA was used as input for NGS library construction. To assess RNA quality, the PreSeq RNA QC Assay using iTaq Universal SYBR Green Supermix (Biorad, Hercules, CA) was performed on all samples during library preparation to generate a measure of the integrity of RNA (in the form of a cycle threshold [Ct] value). Library preparation and RNA QC were performed following the Archer FusionPlex Protocol for Illumina (ArcherDX Inc.). The Archer FusionPlex Solid Tumor Kit was used. Final libraries were diluted $1: 100,000$ and quantified in a $10 \mu \mathrm{L}$ reaction following the Library Quantification for Illumina Libraries protocol and assuming a $200 \mathrm{bp}$ fragment length (KAPA, Wilmington, MA). The concentration of final libraries was around $200 \mathrm{nM}$. Threshold representing the minimum molar concentration for which sequencing can be robustly performed was set at $50 \mathrm{nM}$.

\section{NGS Sequencing and Analysis}

Libraries were diluted to $4 \mathrm{nM}$ and sequenced on a NextSeq sequencer (Illumina, San Diego, CA). The optimal number of raw reads per sample was set to 3 million. Library pools were diluted to $1.6 \mathrm{pM}$ library stock with $20 \% 1.8 \mathrm{pM}$ PhiX and loaded in the NextSeq cartridge.

The fusion and other rearrangement detection algorithm in Archer Analysis relies on the specificity of the genespecific primers used in the amplification steps in the AMP process. The resulting FASTQ files were analyzed using the Archer Analysis software (version 5.1.7; ArcherDX Inc.).

\section{Fluorescence In Situ Hybridization Analysis of RET Break and TRIM27-RET Fusion}

Four-micrometer-thick FFPE sections were placed onto positively charged slides. Hematoxylin and eosinstained slides were examined for determination of areas for cell counting.

The unstained slides were deparaffinized and incubated in the 1× Target Retrieval Solution Citrate pH 6 (Dako, Glostrup, Denmark) at $95^{\circ} \mathrm{C}$ for 40 minutes and subsequently cooled for 20 minutes at room temperature in the same solution. The slides were washed in deionized water for $5 \mathrm{mi}$ nutes and digested in protease solution with Pepsin $(0.5 \mathrm{mg} /$ $\mathrm{mL}$ ) (Sigma Aldrich, St. Louis, MO) in $0.01 \mathrm{M} \mathrm{HCl}$ at $37^{\circ} \mathrm{C}$ for 35 to 60 minutes according to the sample conditions. The slides were then placed into deionized water for 5 minutes, dehydrated in a series of ethanol solution $(70 \%, 85 \%$, and $96 \%$ for 2 min each), and air-dried.

For the detection of $R E T$ rearrangement, factory premixed commercial probe ZytoLight SPEC RET Dual Color Break Apart Probe (ZytoVision GmbH, Bremerhaven, Germany) was used. For the TRIM27-RET dual-fusion detection custom-designed SureFISH probe (Agilent Technologies Inc., Santa Clara) with chromosomal regions chr6:28631476-29131075 and chr10:4335489343849282 was used. Probe mixture for fusion detection was prepared from corresponding probes (each color was delivered in a separated well), deionized water, and LSI Buffer (Vysis/Abbott Molecular) in a 1:1:1:7 ratio, respectively.

An appropriate amount of mixed and premixed probes was applied on specimens, covered with a glass coverslip, and sealed with rubber cement. The slides were incubated in the ThermoBrite instrument (StatSpin/Iris Sample Processing, Westwood, MA) with co-denaturation at $85^{\circ} \mathrm{C} / 8$ minutes and hybridization at $37^{\circ} \mathrm{C} / 16$ hours. The rubber-cemented coverslip was then removed, and the slide was placed in posthybridization wash solution ( $2 \mathrm{xSSC} / 0.3 \% \mathrm{NP}-40)$ at $72^{\circ} \mathrm{C} / 2$ minutes. The slide was airdried in the dark, counterstained with $4^{\prime}, 6^{\prime}$-diamidino2-phenylindole DAPI (Vysis/Abbott Molecular), cover slipped, and immediately examined.

\section{Fluorescence In Situ Hybridization Interpretation}

The sections were examined with an Olympus BX51 fluorescence microscope (Olympus Corporation, Tokyo, Japan) using a $\times 100$ objective and filter sets 
Triple Band Pass (DAPI/SpectrumGreen/SpectrumOrange), Dual Band Pass (SpectrumGreen/SpectrumOrange), and Single Band Pass (SpectrumGreen or SpectrumOrange).

For each probe, 100 randomly selected nonoverlapping tumor cell nuclei were examined for the presence of yellow or green and orange fluorescent signals. As regards break-apart probe, yellow signals were considered negative, and separate orange and green signals were considered as positive; conversely, for fusion probe, yellow signals were considered positive, and separate orange and green signals were considered as negative. Cut-off values for break-apart and fusion probes were set to $>10 \%$ and $20 \%$ of nuclei with chromosomal breakpoint and fusion signals, respectively (mean +3 $\mathrm{SD}$ in normal non-neoplastic control tissues).

\section{RT-PCR Analysis of NCOA4-RET and TRIM27-RET Fusion Transcripts}

Two sample of cDNA prepared by standard reverse transcription procedure using Transcriptor First Strand cDNA Synthesis Kit (RNA input $500 \mathrm{ng}$ ) (Roche Diagnostics, Mannheim, Germany) was added to the reaction mixture consisting of $12.5 \mu \mathrm{L}$ of HotStar Taq PCR Master Mix (QIAgen, Hilden, Germany), 10 pmol of each fusionspecific primer (Table 2), and distilled water up to $25 \mu \mathrm{L}$. The amplification program comprised denaturation at $95^{\circ}$ $\mathrm{C}$ for 14 minutes and then 45 cycles of denaturation at $95^{\circ}$ $\mathrm{C}$ for 1 minute, annealing at a temperature of $55^{\circ} \mathrm{C}$ for 1 minute and extension at $72^{\circ} \mathrm{C}$ for 1 minute. The program was completed by incubation at $72^{\circ} \mathrm{C}$ for 7 minutes.

Successfully amplified PCR products were purified with magnetic particles Agencourt AMPure (Agencourt Bioscience Corporation, A Beckman Coulter Company, Beverly, MA). Products were then bidirectionally sequenced using Big Dye Terminator Sequencing kit (Applied Biosystems, Foster City, CA), purified with magnetic particles Agencourt CleanSEQ (Agencourt Bioscience Corporation), all according to the manufacturer's protocol, and run on an automated sequencer ABI Prism 3130 $\times 1$ (Applied Biosystems) at a constant voltage of $13.2 \mathrm{kV}$ for 11 minutes.

\section{RESULTS}

\section{Molecular Genetic Findings}

Thirty-three cases of IC were analyzed by NGS using the Anchored Multiplex PCR (AMP) chemistry technique (ArcherDX). An NCOA4-RET fusion transcript

TABLE 2. Primers Used for RT-PCR Analysis

\begin{tabular}{ll}
\hline Name of Primer & \multicolumn{1}{c}{ Sequence 5' -3' $^{\prime}$} \\
\hline NCOA4-RET_7-12_F & CCCTTCCTGGAGAAGAGAGG \\
NCOA4-RET_7-12_R & GTACCCTGCTCTGCCTTTCA \\
NCOA4-RET_8-12_F & TACCCAAAAGCAGACCTTGG \\
NCOA4-RET_8-12_R & CGCCTTCTCCTAGAGTTTTCC \\
TRIM27-RET_3-12_F & TGATCGCTCAGCTAGAAGAGAA \\
TRIM27-RET_3-12_R & CCAAGTTCTTCCGAGGGAAT \\
TUT1-ETV5_7-8_F & GTACTCGGCCTAGCCTCCAC \\
TUT1-ETV5_7-8_R & TCTGACATTTGCCGATGGTA \\
KIA1217-RET_14-9_F & CCAGTGGACAAGGTGGAACT \\
KIA1217-RET_14-9_R & AGGTGCCATAGCCAGCTTTA \\
\hline
\end{tabular}

4 | www.ajsp.com joining exon 7 or 8 of the NCOA4 gene and exon 12 of the $R E T$ gene was detected in 11 cases of intercalated duct or hybrid types of IC. A novel TRIM27-RET fusion transcript between exon 3 of TRIM27 and exon 12 of RET was detected in 2 cases with histologic and immunohistochemical features typical of the apocrine variant of IC. Two IC cases with invasion harbored novel fusions TUT1-ETV5 and KIAA1217-RET, respectively. The fusion transcripts were confirmed by RT-PCR and by sequencing. Fluorescence in situ hybridization (FISH) analysis using $R E T$ break-apart probe showed 2 different patterns. One was deemed "negative" when distance criteria of the break-apart probe were used according to interpretation rules, even if a minor separation of signals was seen (cases 1 to 2, 8, 15 to 19, 26, and 29). The other pattern showed a regular break (classic positive pattern) with separation of signals detected in 3 cases (cases 3, 28, and 31). Confirmation using FISH TRIM27-RET fusion probe displayed fusion signals with amplification. Localization of RET, NCOA4, and TRIM27 genes to obtain an explanation of the 2 different patterns in FISH breakapart results is shown in the Figure 1.

A total of $42.4 \%$ of the analyzed cases harbored an RET fusion by NGS. This was less than that in previous reports, ${ }^{5,6}$ but almost one-third of our cases were found not analyzable (NA). By RT-PCR, the fusions NCOA4-RET and TRIM27-RET were confirmed in all NGS-positive cases. The RET break-apart probe and the TRIM27-RET fusion probe successfully detected rearrangement in both cases with TRIM27-RET fusion identified by NGS. Only a narrow separation of the FISH signals for RET rearrangement was obtained, as this rearrangement contains an intrachromosomal inversion. Such "false"-negative FISH results were obtained for all NCOA4-RET cases. The molecular findings are summarized in Tables 3 and 4.

Ten cases of high-grade salivary carcinomas, originally diagnosed as SDC, were analyzed by NGS using the Fusion Plex Solid Tumor and Comprehensive Thyroid and Lung (CTL) kits for Illumina (ArcherDX). The analysis revealed one case harboring an ETV6-NTRK3 fusion, and therefore it was reclassified as a high-grade secretory carcinoma. NGS also detected 5 likely pathogenic mutations in 5 SDC cases (HRAS: c. $182 \mathrm{~A}>\mathrm{G}$ p.Gln61Arg, HRAS: c. $37 \mathrm{G}>\mathrm{C}$ p. Gly13Arg, AKT1: c.49G > A p.Glu17Lys, PTEN c.1003C > $\mathrm{T}$ p.Arg335Ter). Homozygous deletion of locus 9p21 $(C D K N 2 A)$ was detected in one case harboring an HRAS mutation. Two cases presented rearrangement of the $M Y B$ gene, while one case was positive for PLAGl rearrangement. $M D M 2$ amplification was found in one case harboring also a PTEN mutation. The other SDC cases were negative for $M D M 2$ amplification and showed no rearrangement in the genes MYB, MYBL1, NFIB, NTRKI, RET, and PLAG1. Neither NCOA4-RET nor TRIM27-RET fusions were detected in any SDC cases.

\section{Clinical and Histologic Characteristics of the Study Group}

The study series consisted of 33 ICs; 25 neoplasms were pure intraductal in situ lesions, while 8 cases showed apparent 

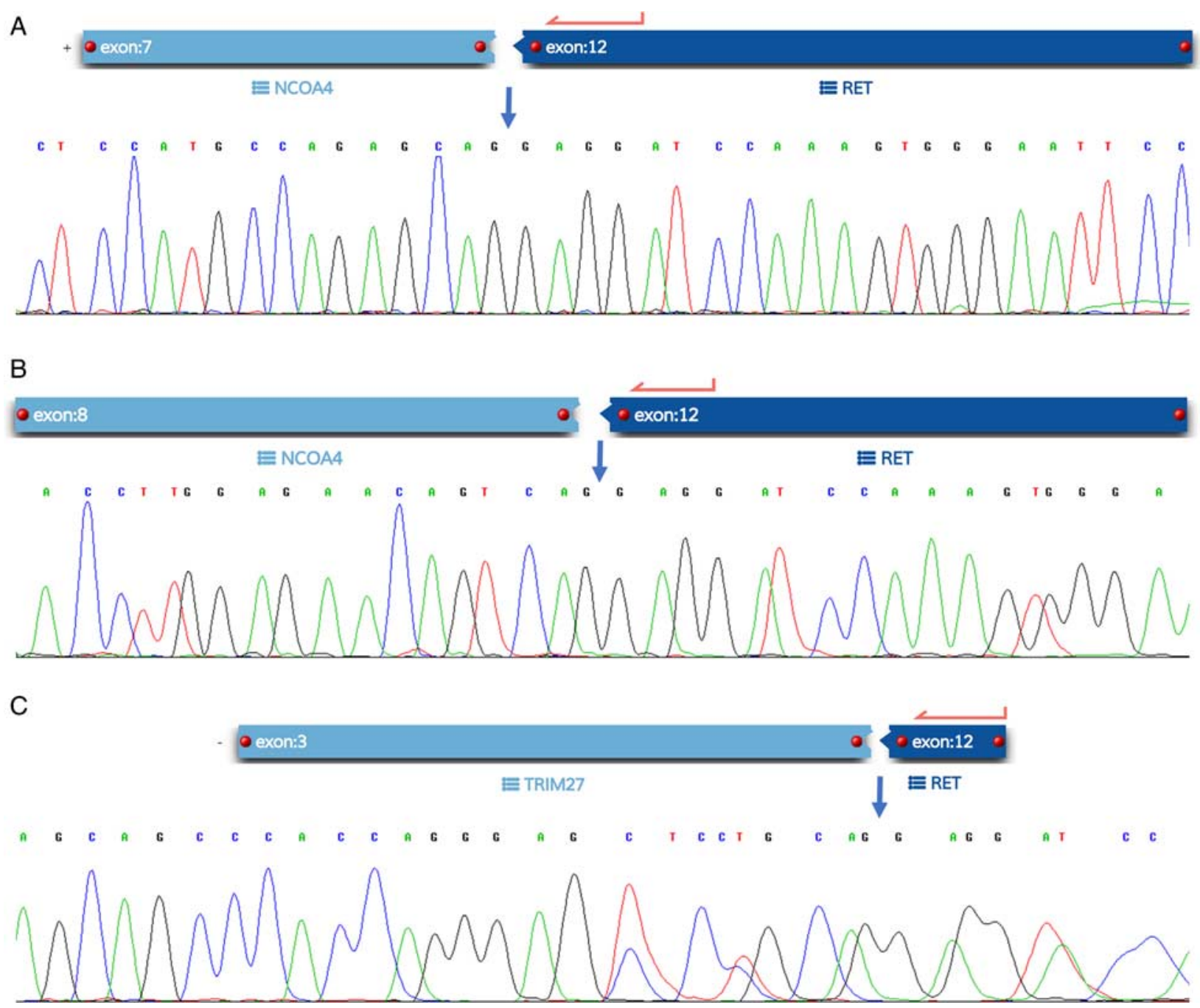

FIGURE 1. A-C, Sanger sequencing analysis of RT-PCR generated fusion transcripts NCOA4-RET (exon joining 7 to 12), NCOA4-RET (exon joining 8 to 12), and TRIM27-RET (exon joining 3 to 12). The arrow shows the fusion position.

invasive growth, in one case with a single metastasis in a periparotideal lymph node. The invasive carcinomas occurred in patients ranging in age from 36 to 64 years (mean: $52.5 \mathrm{y}$ ), and noninvasive IC in patients ranging in age from 17 to 81 years (mean: $56 \mathrm{y}$ ). The most common anatomic site of involvement was the parotid gland $(\mathrm{n}=28)$, and there was 1 case each in the buccal mucosa, the palate, and the submandibular gland. Detailed clinical and histopathologic findings and follow-up information of 33 patients with IC are summarized in Table 4.

\section{Microscopic and Immunohistochemical Features}

Histologically, at low-power magnification, the ICs were well circumscribed and encapsulated in most cases. They were characterized by luminal epithelial proliferations with intercalated duct phenotype arranged mainly in multiple cystic patterns, besides solid and cribriform islands in 24/33 (73\%) (Fig. 2A). Intracystic growth often formed micropapillary structures with anastomosing epithelial tufts, with snouts, decapitation secretion, and apocrine features in 6/33 cases $(18 \%)$ (Fig. 2C). A hybrid intercalated duct and apocrine growth pattern were seen in $4 / 33$ cases $(12 \%)$. Widespread or focal invasive growth was found in 8 cases. In the cases of invasion, we estimate that the invasive component ranged roughly from 10 to $60 \%$ of the total tumor area. While no peritumoral lymphovascular invasion was noted in the IC cases, perineural invasion (Fig. 3A) and metastatic infiltration in a periparotid lymph node were observed in 1 case (Fig. 3B). Invasive areas revealed a compressed fibrotic stroma with desmoplasia. Case 6 was an unusual multinodular invasive low-grade carcinoma of the parotid gland composed of 2 distinctive growth patterns. A large part of the tumor was a low-grade salivary epithelial-myoepithelial carcinoma with apocrine differentiation in both the luminal epithelial and the abluminal myoepithelial cells, and it had a broadly invasive growth pattern. Another section of the same tumor revealed a noninvasive IC, of the intercalated duct type, with intracystic papillary and cribriform features. 
TABLE 3. Detailed Molecular Findings of 33 Cases of Salivary Gland Intraductal Carcinomas

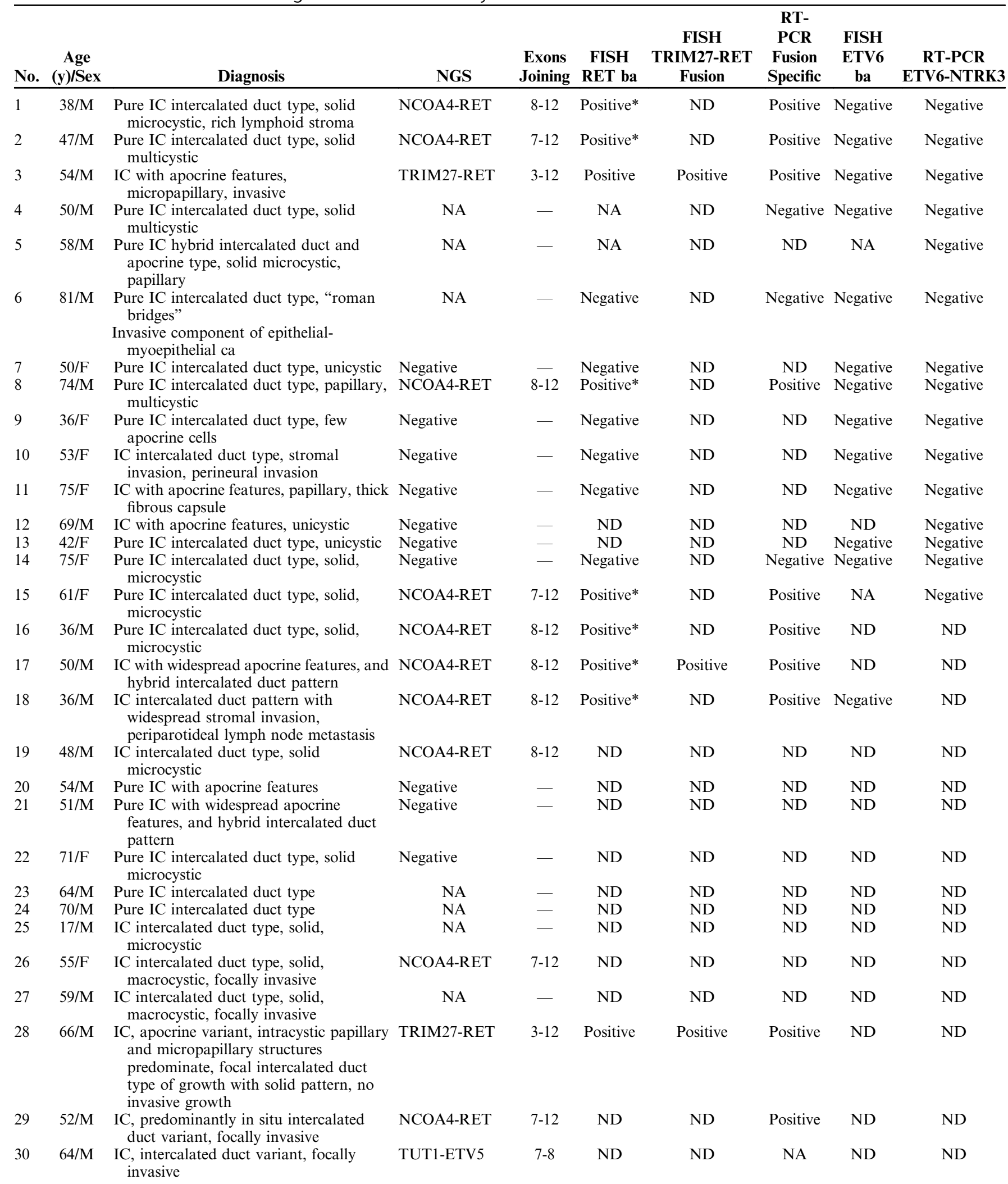


TABLE 3. (continued)

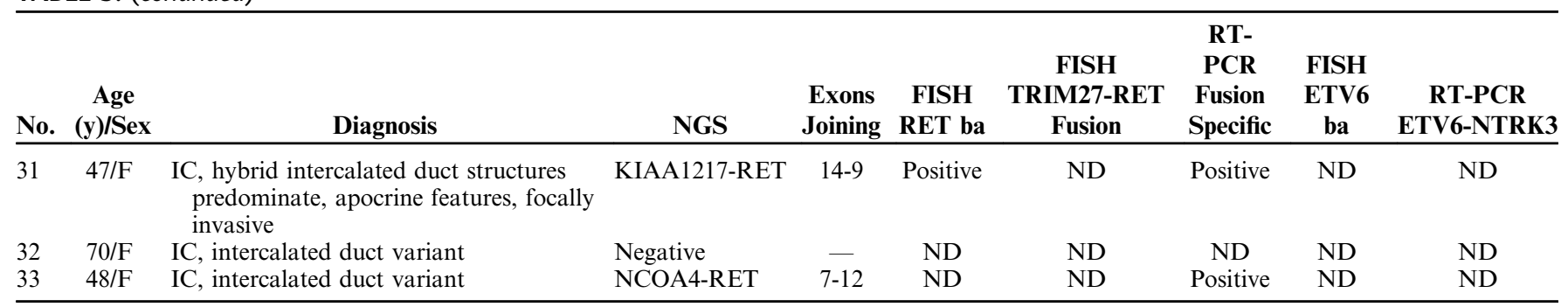

Cases 1 to 17 were published in Skálová et al. ${ }^{6}$

* Cases showed an RET FISH signal pattern indicative of an inversion/rearrangement (in one allele a gap between orange and green signals was observed; hovewer, this gap did not reach internal cutoff ( $\geq 2$ signal diameters apart).

$\mathrm{F}$ indicates female; $\mathrm{M}$, male; NA, not analyzable; ND, not done.

On high-power magnification, all cases showed bland cytologic features, with tumor cells ranging from small to medium size. They had indistinct cell borders and round or ovoid nuclei with dark condensed or finely dispersed chromatin and large pale to eosinophilic cytoplasm. None of the cases in this study had an oncocytic morphology, although an oncocytic variant has been described in the literature. ${ }^{15} \mathrm{Mi}$ toses were inconspicuous. In all 8 IC cases with invasive growth, we have seen compressed sclerotic fibrosis representing apparent desmoplastic reaction around the invasive foci of the tumors (Fig. 3C). These invasive ICs did not have higher MIB1 indices. Proliferative activity in ICs was generally low, with a mean MIB1 index $<5 \%$ (ranging from $1 \%$ to $10 \%$ ). No difference was seen between invasive and noninvasive ICs.

The tumor islands of IC were surrounded by a layer of myoepithelial cells, as evidenced by the immunohistochemical expression of p63, calponin, and/or CK 14 (all of which were negative in luminal epithelial tumor cells) in a continuous abluminal pattern in $14 / 33$ cases $(43 \%)$ (Fig. 2B). A partly discontinuous immunostaining for p63 was seen in most cases (Fig. 3D). All IC cases assessed as completely intraductal were examined in total and stained with antibodies to calponin and p63/p40. Thus, hematoxylin and eosin-based information was verified by continuous immunostains corresponding to intact myoepithelial cell layers. In ICs with apocrine and mixed hybrid growth patterns (10/33 cases; $30 \%$ ), most tumor cells were positive for nuclear AR (Fig. 2D). Diffuse and strong coexpression of S100 protein, mammaglobin, and SOX10 was detected in 24/33 cases $(73 \%)$. Pure apocrine ICs were positive for AR and negative for S100 protein/SOX10 and mammaglobin in 4 cases, while, in one case, AR was strongly expressed together with S100 protein/SOX10 and mammaglobin.

\section{DISCUSSION}

The term "intraductal carcinoma" of the salivary gland was first introduced in 1983 by Chen ${ }^{10}$ in a description of a single case arising in the minor salivary gland of the oral cavity. Subsequently, several case reports and small series of the same tumor entity were published with various names, including "low-grade salivary duct carcinoma" 2,8 and "low-grade cribriform cystadenocarcinoma." The latter designation was introduced by the editors of the 2005 WHO Classification of Head and Neck Tumours, although this term had never appeared in the literature before that point. ${ }^{3}$ This term referred to cystic and solid structures formed by intraductal epithelial proliferation of cells resembling intercalated duct cells and structures reminiscent of atypical ductal hyperplasia and ductal carcinoma in situ of the breast. In the 2017 WHO Classification of Head and Neck Tumours, this tumor is regarded as an IC. ${ }^{1}$ IC has been surrounded by controversy since its description not only because of variable terminologies but primarily because of the uncertain relationship to salivary duct carcinoma. ${ }^{2,3,8,11}$ In typical cases, IC is a noninvasive low-grade carcinoma composed of bland neoplastic cells positive for S100 protein, in sharp contrast to those features seen in SDC. SDC is defined by the 2017 WHO classification as an aggressive epithelial malignancy resembling high-grade mammary ductal carcinoma. It is composed of pleomorphic S100 protein-negative and AR-positive neoplastic cells. ${ }^{16}$ Therefore, these 2 lesions are generally accepted as distinct and separate entities. ${ }^{5,6,13,14}$ However, the literature describes occasional well-documented low-grade ICs with widespread invasive growth. 2,3,5,6 Even more confusing are rare cases of true high-grade SDC reported as purely "in situ lesions." 11 Moreover, IC cases with hybrid intercalated duct and apocrine features,, 7 oncocytic features, ${ }^{15}$ and pure apocrine features with diffuse AR-positive and S100 protein/ mammaglobin and/or SOX10-negative immunoprofile, ${ }^{4,6}$ have been described.

In recent years, many salivary gland low-grade carcinomas have been found to demonstrate characteristic tumor type-specific chromosomal rearrangements, such as ETV6-NTRK3 and ETV6-RET, in secretory carcinoma (also known as mammary analogue secretory carcinoma), 17,18 EWSRI-ATFI and EWSRI-CREM in hyalinizing clear cell carcinoma of minor salivary glands, ${ }^{19,20}$ CRTC1-MAML2 and CRTC3-MAML2 in mucoepidermoid carcinoma, ${ }^{21}$ ARIDIA-PRKD1 and variant PRKD1, PRKD2, and $P R K D 3$ fusions in cribriform adenocarcinoma of minor salivary glands and $P R K D 1$ somatic mutations in polymorphous adenocarcinoma, ${ }^{22-24}$ and HTN3-MSANTD3 in a subset of acinic cell carcinoma of the salivary gland. ${ }^{25}$ The growing list of gene fusion-positive salivary carcinomas 


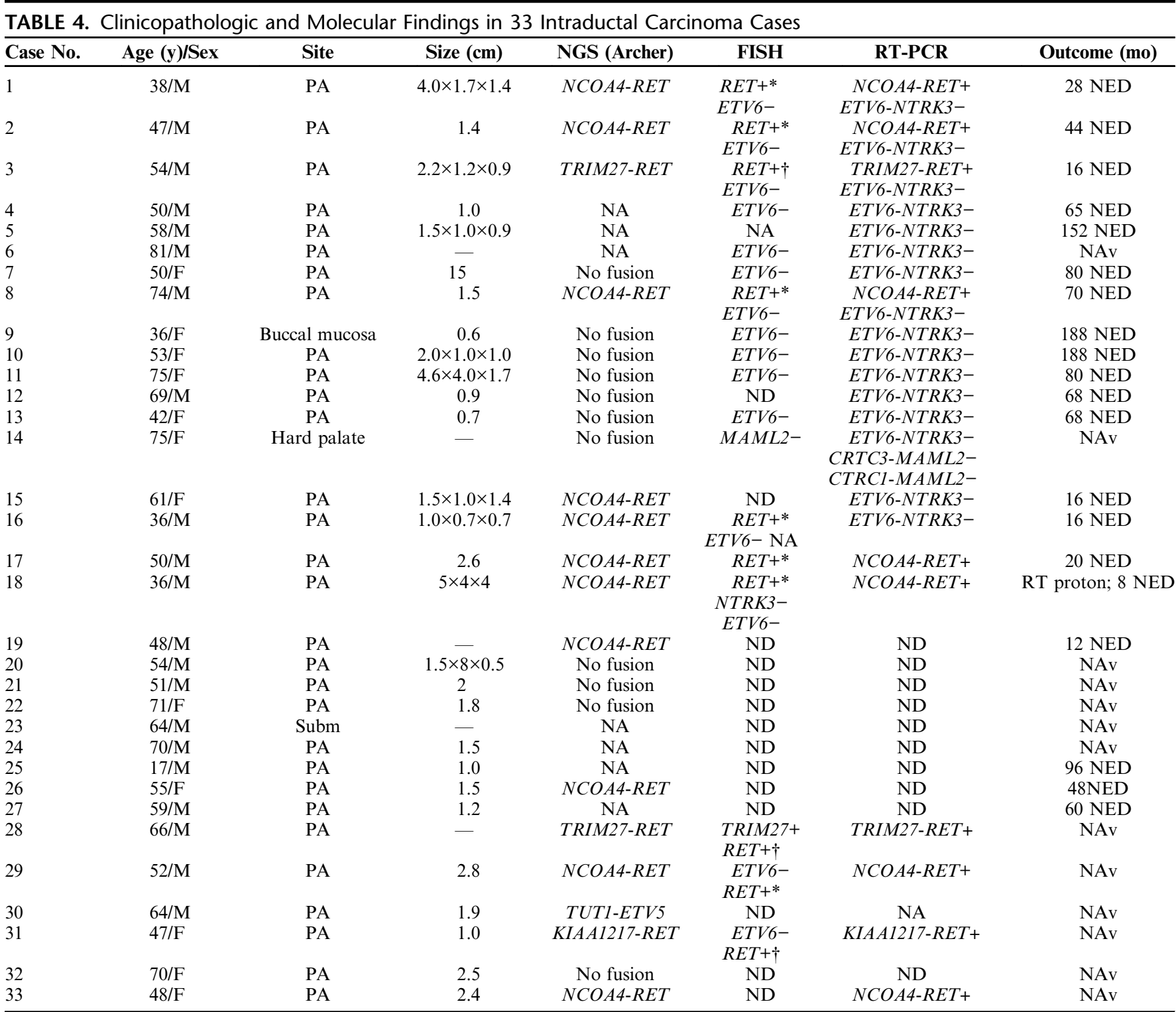

* Cases showed an RET FISH signal pattern indicative of an inversion/rearrangement.

$\dagger$ Cases 3, 28, and 31 showed a classic break-apart signal pattern.

F indicates female; M, male; NA, not anlyzable; NAv, not available; ND, not done; NED, no evidence of disease; PA, parotid gland; subm, submandibular gland; $\mathrm{RT}$ proton, radiotherapy in proton center.

includes IC with an NCOA4-RET fusion reported recently. 5,6 In addition, we have described a subset of IC with apocrine morphology harboring a novel TRIM27-RET fusion. ${ }^{6}$ Interestingly, one study using RNA sequencing has revealed NCOA4-RET fusions in 2 cases of salivary gland neoplasms classified as SDC on the basis of expression of AR. ${ }^{13}$ Both NCOA4-RET translocated neoplasms progressed in spite of undergoing concurrent chemoradiation, combination chemotherapy, and dual androgen deprivation therapy. Both patients, however, benefited from RET-targeted therapy. ${ }^{13}$ This would be a challenging finding, but the neoplasm described as NCOA4-RET-translocated SDC, and depicted in their Figure $3 \mathrm{~B}$ is morphologically not compatible with
SDC. ${ }^{13}$ No reference to SOX10, mammaglobin, or S100 protein was made, while their Figure $3 \mathrm{~B}$ shows only focal AR staining. Therefore, it is not clear whether these $R E T$-translocated neoplasms are true SDCs. ${ }^{13}$ It is the opinion of the authors that they most likely represented a hybrid apocrine/intercalated variant of IC.

None of the above-cited gene fusions were documented in SDC cases of the previous study ${ }^{5}$ or in this current series. In view of the tumor type-specific rearrangements described in salivary neoplasms so far, it is not likely that multiple separate salivary tumor types would have the same NCOA4-RET and RET rearrangements. Thus, NCOA4-RET and TRIM27-RET gene 

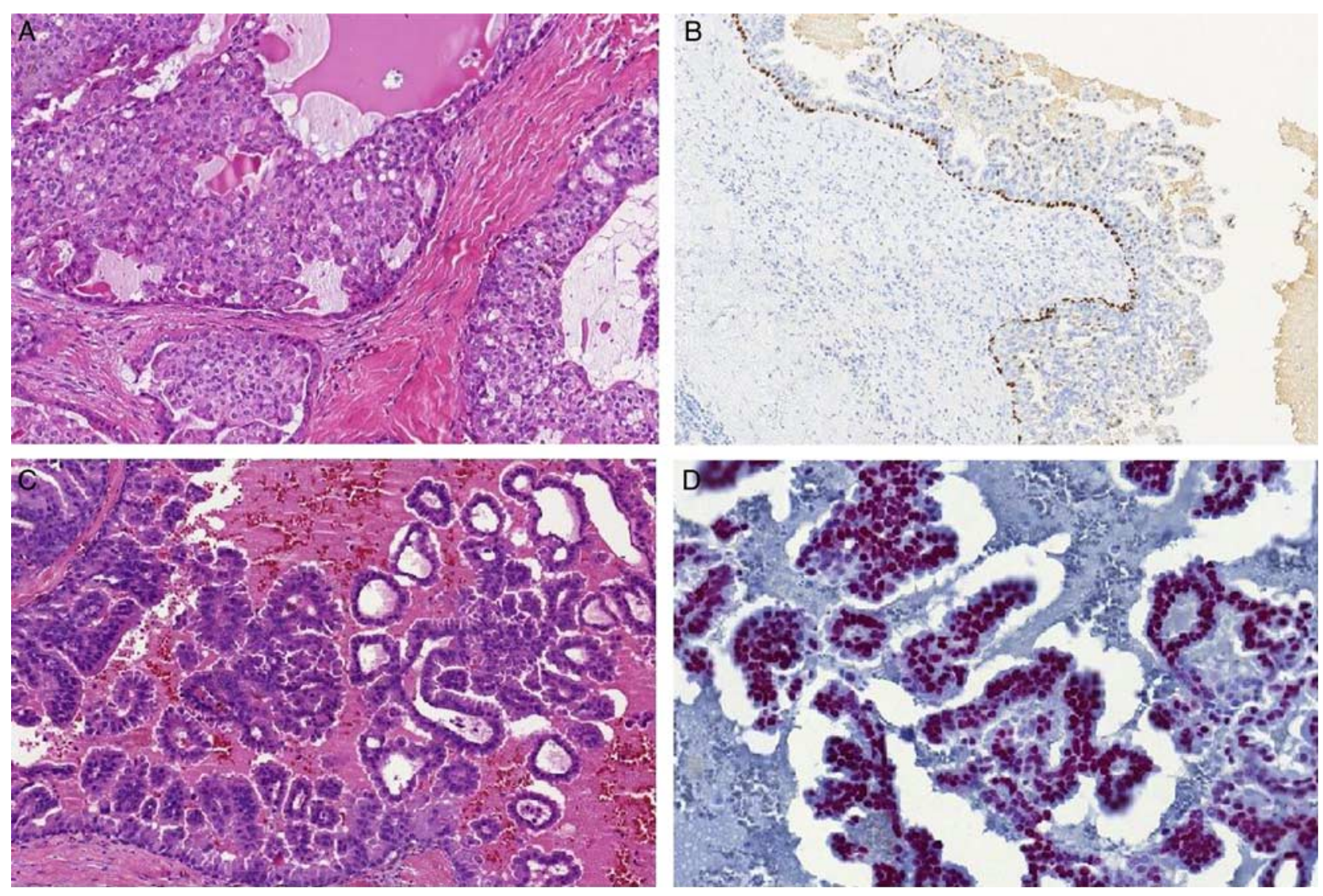

FIGURE 2. IC, intercalated duct variant $(A, B)$, and apocrine variant $(C, D)$. IC is characterized by luminal epithelial proliferations, with intercalated duct phenotype arranged in multiple cystic patterns (A), with the intact myoepithelial abluminal layer decorated by 063 protein in immunohistochemical staining (B). An apocrine variant of IC shows intracystic papillary structures composed of tumor cells exhibiting abundant eosinophilic cytoplasm with apical snouts of decapitation secretion (C) and androgen receptors in immunohistochemical staining (D).

fusions appear to present a rather specific marker of the intercalated duct and apocrine variants of IC, respectively, including cases of widely invasive and metastasizing neoplasms. Therefore, the 2017 WHO Classification term "intraductal carcinoma" is confusing and should be replaced by a more appropriate and accurate one. We propose the designation "intercalated duct carcinoma, noninvasive (or invasive)" for neoplasms formed by intraductal epithelial proliferation composed of cells resembling intercalated duct cells with an S100 protein/ SOX10 and mammaglobin-positive and AR-negative immunoprofile, and an intact or focally disrupted abluminal myoepithelial cell layer. Thus, the intercalated duct carcinoma represents a line of differentiation toward intercalated duct features, but, in some cases, ICs may show oncocytic, ${ }^{15}$ apocrine, or clear cell features. The neoplasms characterized by apocrine morphology, and an AR-positive and S100 protein/SOX10-negative immunoprofile harboring the TRIM27-RET gene fusion, should be designated "intercalated duct carcinoma, with apocrine features." Interestingly, we observed one case of pure intercalated duct IC associated with invasive low-grade epithelial-myoepithelial carcinoma (EMCa).
Fittingly, EMCa also recapitulates the intercalated duct phenotype, ${ }^{26,27}$ and has been suggested to differentiate in that direction. Moreover, the reported observation of background intercalated duct hyperplasia in some cases of EMCa suggests the possibility of an intercalated duct precursor lesion. ${ }^{28,29}$

In contrast to IC, the well-established term "salivary duct carcinoma" should be reserved for widely invasive high-grade malignancies and should be classified according to previously reported criteria. ${ }^{14}$ SDCs have shown a number of genomic alterations including HER2-neu gene amplification, and hotspot PIK3CA and HRAS mutations and many others, not present in ICs. ${ }^{16,30-32}$

A novel finding in our series was a case of widely invasive intercalated duct-type IC, with a single lymph node metastasis that revealed an NCOA4-RET fusion transcript. Two IC cases with invasive growth harbored novel fusions TUT1-ETV5 and KIAA1217-RET, respectively. Finally, NCOA4-RET and TRIM27-RET, TUT1-ETV5, and KIAA1217-RET were not detected by NGS in any of the 10 SDCs studied as a comparison group. The molecular distinction reported herein between IC and SDC supports the idea that they represent separate malignant salivary tumor 

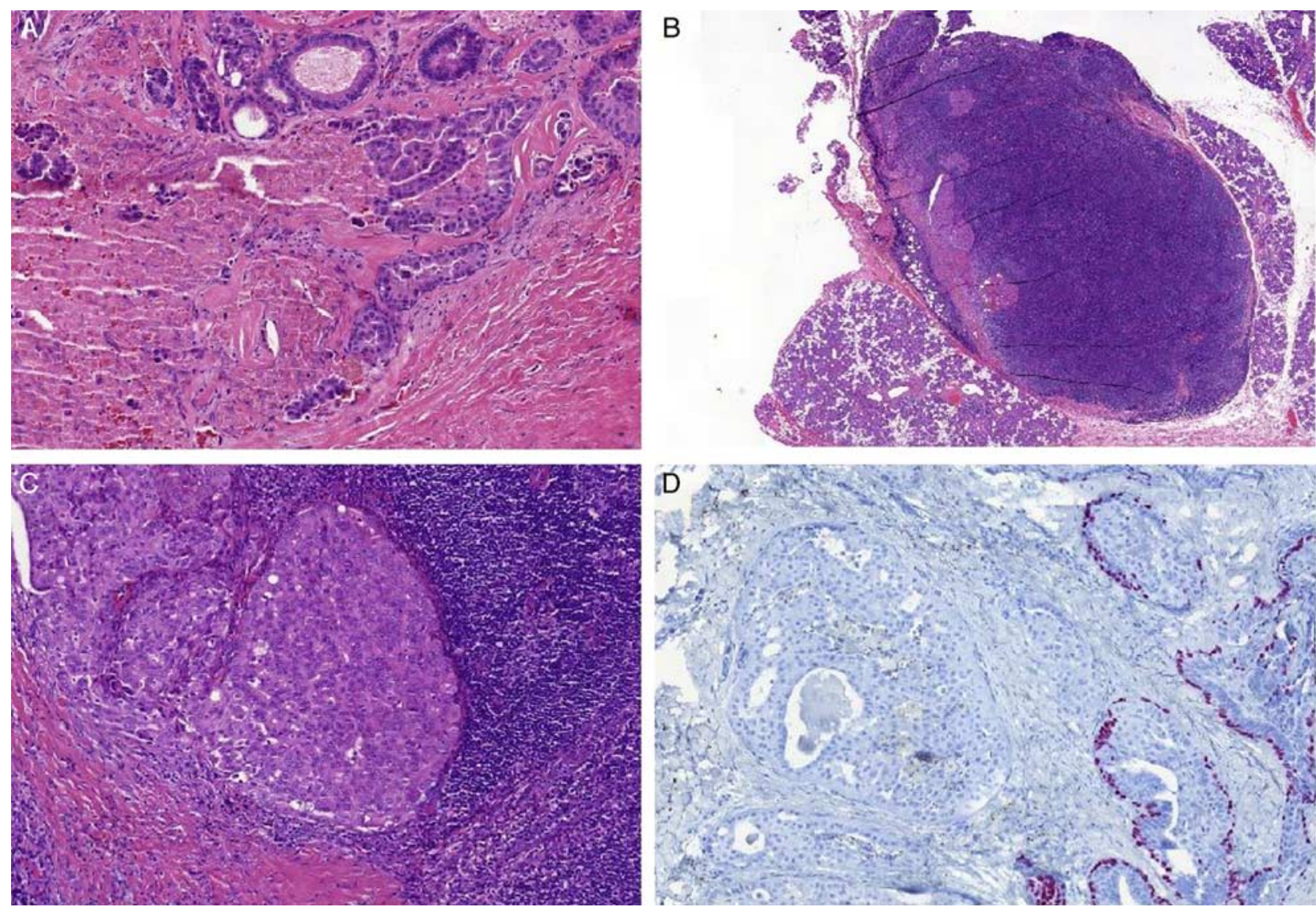

FIGURE 3. Invasive IC (A-D). The invasive growth pattern is seen in IC with perineural infiltration (A) and metastatic invasion in periparotideal lymph node $(B, C)$. The discontinuous myoepithelial layer is seen in immunohistochemical staining with p63 antibody (D).

entities, and should be considered separately in future classifications.

In summary, recurrent rearrangements involving the RET gene were identified in a subset of invasive and metastasizing IC, with intercalated duct and apocrine variants resulting in NCOA4-RET and TRIM27-RET fusions, respectively. Patients with locally invasive, advanced, and metastatic RET-rearranged tumors could benefit from targeted therapy using $R E T$-inhibitors. ${ }^{33-36}$ However, the presence of oncogenic rearrangements cannot be predicted reliably by tumor morphology only. Therefore, we propose that salivary gland cancers should be tested in a screening program for targetable RET alterations, if available. Finally, our study indicates that ICs can also manifest significant invasive growth, and, consequently, a designation of these tumors as ICs seems inappropriate by conventional histopathologic rules. The authors suggest that IC should be redesignated as intercalated duct carcinoma, noninvasive or invasive.

\section{ACKNOWLEDGMENTS}

The authors thank Prof. Cécile Badual, MD, PhD, Paris, France (case 4), Prof. Jan Laco, MD, PhD, Hradec Králové, Czech Republic (cases 7, 12 to 13), Petr Hrabal,
MD, Prague, Czech Republic (case 10), Prof. Nina Zidar, MD, PhD, Ljubljana, Slovenia (case 14), and Vera Fischerova, MD, Ceske Budejovice, Czech Republic (case 18) for submitting their cases, and thus contributing in the study.

\section{REFERENCES}

1. Loening T, Leivo I, Simpson RHW, et al. Intraductal carcinoma. In: El-Naggar A, Chan JKC, Grandis JR, Takata T, Slootweg PJ, eds. World Health Organization (WHO) Classification of Head and Neck Tumours, 4th ed. Lyon, France: IARC Press; 2017:170-171.

2. Delgado R, Klimstra D, Albores-Saavedra J. Low grade salivary duct carcinoma. A distinctive variant with a low grade histology and a predominant intraductal growth pattern. Cancer. 1996;78:958-967.

3. Brandwein-Gensler MS, Gnepp DR. WHO classification of tumours. In: Barnes L, Eveson JW, Reichart P, Sidransky D, eds. Pathology and Genetics of Head and Neck Tumours. Lyon: IARC Press; 2005: 233.

4. Weinreb I, Tabanda-Lichauco R, Van der Kwast T, et al. Low-grade intraductal carcinoma of salivary gland: report of 3 cases with marked apocrine differentiation. Am J Surg Pathol. 2006;30: 1014-1021.

5. Weinreb I, Bishop JA, Chiosea SI, et al. Recurrent RET gene rearrangements in intraductal carcinomas of salivary gland. $A m J$ Surg Pathol. 2018;42:442-452.

6. Skálová A, Vanecek T, Uro-Coste E, et al. Molecular profiling of salivary gland intraductal carcinoma revealed a subset of tumors harboring NCOA4-RET and novel TRIM27-RET fusions: a report of 17 cases. Am J Surg Pathol. 2018;42:1445-1455. 
7. Lu H, Graham RP, Seethala R, et al. Intraductal carcinoma of salivary glands harboring TRIM27-RET fusion with mixed low grade and apocrine types. Head Neck Pathol. 2019. [Epub ahead of print].

8. Brandwein-Gensler M, Hille J, Wang BY, et al. Low-grade salivary duct carcinoma: description of 16 cases. Am J Surg Pathol. 2004:28:1040-1044.

9. Bahrami A, Perez-Ordonez B, Dalton JD, et al. An analysis of PLAG1 and $H M G A 2$ rearrangements in salivary duct carcinoma and examination of the role of precursor lesions. Histopathology. 2013;63:250-262.

10. Chen KT. Intraductal carcinoma of the minor salivary gland. J Laryngol Otol. 1983;97:189-191.

11. Simpson RH, Desai S, Di Palma S. Salivary duct carcinoma in situ of the parotid gland. Histopathology. 2008;53:416-425.

12. Cheuk W, Miliauskas JR, Chan JK. Intraductal carcinoma of the oral cavity: a case report and a reappraisal of the concept of pure ductal carcinoma in situ in salivary duct carcinoma. Am J Surg Pathol. 2004;28:266-270.

13. Wang K, Russell JS, McDermott JD, et al. Profiling of 149 salivary duct carcinomas, carcinoma ex pleomorphic adenomas, and adenocarcinomas, not otherwise specified reveals actionable genomic alterations. Clin Cancer Res. 2016;22:6061-6068.

14. Simpson RHW. Salivary duct carcinoma: new developmentsmorphological variants including pure in situ high grade lesions; proposed molecular classification. Head Neck Pathol. 2013;7:S48-S58.

15. Nakaguro M, Urano M, Suzuki H, et al. Low-grade intraductal carcinoma of the salivary gland with prominent oncocytic change: a newly described variant. Histopathology. 2018;73:314-320.

16. Nagao T, Licitra L, Loening $T$, et al. Salivary duct carcinoma. In: El-Naggar A, Chan JKC, Grandis JR, Takata T, Slootweg PJ, eds. World Health Organization (WHO) Classification of Head and Neck Tumours, 4th ed. Lyon, France: IARC Press; 2017:173-174.

17. Skálová A, Vanecek T, Sima R, et al. Mammary analogue secretory carcinoma of salivary glands, containing the ETV6-NTRK3 fusion gene: a hitherto undescribed salivary gland tumor entity. Am J Surg Pathol. 2010;34:599-608.

18. Skálová A, Vanecek T, Martinek P, et al. Molecular profiling of mammary analog secretory carcinoma revealed a subset of tumors harboring a novel ETV6-RET translocation: report of 10 cases. Am J Surg Pathol. 2018;42:234-246.

19. Antonescu CR, Katabi N, Zhang L, et al. EWSR1-ATFl fusion is a novel and consistent finding in hyalinizing clear-cell carcinoma of salivary gland. Genes Chromosomes Cancer. 2011;50:559-570.

20. Chapman E, Skálová A, Ptakova N, et al. Molecular profiling of hylinizing clear cell carcinoma revealed a subset of tumors harboring a novel EWSR1-CREM fusion: report of three cases. Am J Surg Pathol. 2018;42:1182-1189.

21. Jee KJ, Persson M, Heikinheimo K, et al. Genomic profiles and CRTC1-MAML2 fusion distinguish different subtypes of mucoepidermoid carcinoma. Mod Pathol. 2013;26:213-222.
22. Skálová A, Sima R, Kaspirkova-Nemcova J, et al. Cribriform adenocarcinoma of minor salivary gland origin principally affecting the tongue: characterization of new entity. Am J Surg Pathol. 2011;35:1168-1176.

23. Weinreb I, Zhang L, Tirunagari LM, et al. Novel PRKD gene rearrangements and variant fusions in cribriform adenocarcinoma of salivary gland origin. Genes Chromosomes Cancer. 2014;53: 845-856.

24. Weinreb I, Piscuoglio S, Martelotto LG, et al. Hotspot activating PRKD1 somatic mutations in polymorphous low-grade adenocarcinomas of the salivary glands. Nat Genet. 2014;46:1166-1169.

25. Andreasen S, Varma S, Barasch N, et al. The HTN3-MSANTD3 fusion gene defines a subset of acinic cell carcinoma of the salivary gland. Am J Surg Pathol. 2019;43:489-496.

26. Corio RL, Sciubba JJ, Brannon RB, et al. Epithelial-myoepithelial carcinoma of intercalated duct origin. A clinicopathologic and ultrastructural assessment of sixteen cases. Oral Surg Oral Med Oral Pathol. 1982;53:280-287.

27. Luna MA, Ordonez NG, Mackay B, et al. Salivary epithelial myoepithelial carcinomas of intercalated ducts: a clinical, electron microscopic, and immunocytochemical study. Oral Surg Oral Med Oral Pathol. 1985;59:482-490.

28. Chetty R. Intercalated duct hyperplasia: possible relationship to epithelial-myoepithelial carcinoma and hybrid tumours of salivary gland. Histopathology. 2000;37:260-263.

29. Di Palma S. Epithelial-myoepithelial carcinoma with co-existing multifocal intercalated duct hyperplasia of the parotid gland. Histopathology. 1994;25:494-496.

30. Shimura T, Tada Y, Hirai H, et al. Prognostic and histogenetic roles of gene alteration and the expression of key potentially actionable targets in salivary duct carcinomas. Oncotarget. 2018;9:1852-1867.

31. Dalin MG, Desrichard A, Katabi N, et al. Comprehensive molecular characterization of salivary duct carcinoma reveals actionable targets and similarity to apocrine breast cancer. Clin Cancer Res. 2016;22: 4623-4633

32. Khoo TK, Yu B, Smith JA, et al. Somatic mutations in salivary duct carcinoma and potential therapeutic targets. Oncotarget. 2017;8: 75893-75903

33. Roskoski R Jr, Sadeghi-Najad A. Role of RET protein-tyrosine kinase inhibitors in the treatment $R E T$-driven thyroid and lung cancers. Pharmacol Res. 2018;128:1-17.

34. Drilon A, Hu Zishuo I, Lai GGY, et al. Targeting RET-driven cancers: lessons from evolving preclinical and clinical landscapes. Nat Rev Clin Oncol. 2018;15:151-167.

35. Li GG, Somwar R, Joseph J, et al. Antitumor activity of RXDX-105 in multiple cancer types with RET rearrangements or mutations. Clin Cancer Res. 2017;23:2981-2990.

36. Sabari JK, Siau ED, Drilon A. Targeting RET-rearranged lung cancers with multikinase inhibitors. Oncoscience. 2017;4:23-24. 